\title{
The Spectrum of a Quasiperiodic Schrödinger Operator
}

\section{András Sütő*}

Institut de Physique Théorique, Université de Lausanne, CH-1015 Lausanne, Switzerland

\begin{abstract}
The spectrum $\sigma(H)$ of the tight binding Fibonacci Hamiltonian $\left(H_{m n}=\delta_{m, n+1}+\delta_{m+1, n}+\delta_{m, n} \mu v(n), v(n)=\chi_{\left[-\omega^{3}, \omega^{2}[\right.}((n-1) \omega), 1 / \omega\right.$ is the golden number) is shown to coincide with the dynamical spectrum, the set on which an infinite subsequence of traces of transfer matrices is bounded. The point spectrum is absent for any $\mu$, and $\sigma(H)$ is a Cantor set for $|\mu| \geqq 4$. Combining this with Casdagli's earlier result, one finds that the spectrum is singular continuous for $|\mu| \geqq 16$.
\end{abstract}

Consider the discrete Schrödinger operator $H$ acting on doubly infinite sequences $(\ldots, \psi(-1), \psi(0), \psi(1), \ldots)$, and defined by $(H \psi)(n)=\psi(n+1)+\psi(n-1)+\mu v(n) \psi(n)$ with the potential

$$
v(n)=\chi_{\left[-\omega^{3}, \omega^{2}[((n-1) \omega)\right.}
$$

Here $\omega=(\sqrt{5}-1) / 2$, and $\chi_{I}$ is the characteristic function of the interval $I . H$ is a bounded self-adjoint operator on $l^{2}(\mathbb{Z})$; we are interested in its spectrum. This problem was originally proposed by Kohmoto et al., [1] and Ostlund et al., [2]. Mathematical properties of the sequence (1) were discussed earlier [3], and $v(n)$ appeared also in some models of dissipative systems $[4,5]$. The interest in this particular, nongeneric example of a quasiperiodic Schrödinger operator is explained by its connection with a simple dynamical system whose evolution can be studied with relative ease, so that one may hope for detailed numerical and rigorous results. Moreover, the spectrum of $H$ has long been suspected to be singular continuous, irrespectively of the value of $\mu$.

Let $F_{0}=F_{1}=1, F_{n+1}=F_{n}+F_{n-1}$. For any solution $\psi$ of $H \psi=E \psi$ one can write

where

$$
\Psi_{N}=T_{N} T_{N-1} \ldots T_{1} \Psi_{0}, \quad N \geqq 1
$$

$$
\Psi_{N}=\left(\begin{array}{c}
\psi(N+1) \\
\psi(N)
\end{array}\right), \quad T_{N}=\left(\begin{array}{cr}
E-\mu v(N) & -1 \\
1 & 0
\end{array}\right) \text {. }
$$

* On leave from the Central Research Institute for Physics, Budapest, Hungary 
In particular,

$$
\Psi_{F_{n}}=M(n) \Psi_{0}, \quad M(n)=T_{F_{n}} T_{F_{n}-1} \ldots T_{1}, \quad n \geqq 1 .
$$

The trace, $2 x_{n}$, of $M(n)$ was shown [1] to satisfy the recursion relation $x_{n}=$ $2 x_{n-1} x_{n-2}-x_{n-3}$. Due to the repeated multiplication, this sequence is likely to diverge for almost all initial conditions, i.e., $\mu$ and $E$. Indeed, this was found numerically [1] and by a renormalization group argument [6]. In a recent rigorous study [7] Casdagli proved that

$$
B_{\infty}=\left\{E \in \mathbb{R} \mid\left\{x_{n}\right\} \text { is bounded }\right\}
$$

is a Cantor set of zero Lebesgue measure for $|\mu| \geqq 16$. However, the relation between the dynamical spectrum $B_{\infty}$, and $\sigma(H)$, the spectrum in the usual sense, remained, so far, unclarified. In the present note we show

Theorem 1. For any real $\mu, \sigma(H)=B_{\infty}$, and the point spectrum is absent.

Remark. Observe that the golden number is in the set of zero measure to which the general result [10], excluding localization, does not apply.

This theorem together with Casdagli's finding implies that the spectrum is purely singular continuous for $|\mu| \geqq 16$. Concerning the Cantor property (without $\lambda(\sigma(H))=0)$, the threshold for $\mu$ can be improved:

Theorem 2. $\sigma(H)$ is a Cantor set for $|\mu| \geqq 4$.

These results are proved below through the use of a series of Propositions. At first, we recollect several properties of the potential (1); most of them were exploited in refs $1-7$.

\section{Proposition 1.}

(i) We have

$$
v(n)=[(n+1) \omega]-[n \omega],
$$

where $[x]=\max \{m \in \mathbb{Z} \mid m \leqq x\}$, and hence

Moreover,

$$
v(-n)=v(n-1), \quad n \geqq 2 .
$$

and

$$
\begin{aligned}
v\left(F_{2}+1\right) & =v(1), \\
v\left(F_{n}+l\right) & =v(l), \quad n \geqq 3, \quad 1 \leqq l \leqq F_{n}
\end{aligned}
$$

$$
v\left(-F_{2 n}+l\right)=v(l), \quad n \geqq 1, \quad 1 \leqq l \leqq F_{2 n+1} .
$$

(ii) Let $M(n)$ be the matrices defined by (1)-(3), $x_{n}=\frac{1}{2} \operatorname{Tr} M(n), n \geqq 1$. Then

and

$$
M(n+2)=M(n) M(n+1), \quad n \geqq 1,
$$

$$
x_{n+2}=2 x_{n+1} x_{n}-x_{n-1}, \quad n \geqq 0,
$$

if $x_{0}=\frac{1}{2} \operatorname{Tr} T_{2}$ and $x_{-1}=1$. Moreover,

$$
I=x_{n+1}^{2}+x_{n}^{2}+x_{n-1}^{2}-2 x_{n+1} x_{n} x_{n-1}-1
$$

is independent of $n$, and hence $I=\left(x_{1}-x_{0}\right)^{2}=\mu^{2} / 4$. 
(iii) $\Psi_{-N-2}=T_{N}^{-1} \ldots T_{1}^{-1} \Psi_{-2}$ for $N \geqq 1$. In particular, let $L(n)=$ $\left(T_{1} T_{2} \ldots T_{F_{n}}\right)^{-1}$ and $y_{n}=\frac{1}{2} \operatorname{Tr} L(n)$, then $\Psi_{-F_{n}-2}=L(n) \Psi_{-2}, y_{n}=x_{n}$ for $n \geqq 1$.

Proof. To see (4) use $\omega^{2}=1-\omega$ and $-\omega^{3}=1-2 \omega$ :

$$
\begin{aligned}
\chi_{\left[-\omega^{3}, \omega^{2}[\right.}((n-1) \omega)=1 & \Leftrightarrow \exists m \in \mathbb{Z}: m-2 \omega \leqq(n-1) \omega<m-\omega \\
& \Leftrightarrow \exists m: n \omega<m \leqq(n+1) \omega \Leftrightarrow[(n+1) \omega]-[n \omega]=1 .
\end{aligned}
$$

The equalities (6) and (7) hold because $F_{n-1} / F_{n}$ is a best approximation to $\omega$ (see, e.g., $[8])$, i.e.,

$$
\left|F_{n} \omega-F_{n-1}\right|=\operatorname{dist}\left(F_{n} \omega, \mathbb{Z}\right)<\operatorname{dist}(l \omega, \mathbb{Z})=\operatorname{dist}(-l \omega, \mathbb{Z}), \quad 1 \leqq l<F_{n} .
$$

The borders of the intervals for $l$ are checked directly by applying $F_{n} \omega=F_{n-1}+$ $(-1)^{n} \omega^{n+1}$. Equation (8) is a consequence of (6), (9) follows from (8) and $\operatorname{det} M(n)=1$, and (10) can be verified by using (9). To prove (iii), apply the reflection symmetry (5) and the property (6). These yield $L(n+2)=L(n) L(n+1)$; since $\operatorname{det} L(n)=1$, one gets $y_{n+3}=2 y_{n+2} y_{n+1}-y_{n}$ for $n \geqq 1 . y_{n}=x_{n}$ can be checked for $n=1,2,3$, and it follows from the recursion for $n \geqq 4$.

The properties (5) and (6) make $v(n)$ similar to a Gordon potential [9]. This suggests the use of the following lemma.

Lemma 1. Let $B$ be a $2 \times 2$ matrix, $\operatorname{det} B=1$. Then

$$
\max \left\{|\operatorname{Tr} B| \cdot\|B x\|,\left\|B^{2} x\right\|\right\} \geqq \frac{1}{2}\|x\|,
$$

for any $x \in \mathbb{C}^{2}$.

Proof. Apply the characteristic equation $B^{2}-\operatorname{Tr} B \cdot B+1=0$ to the vector $x$, and take the norm. This yields

$$
|\operatorname{Tr} B| \cdot\|B x\|+\left\|B^{2} x\right\| \geqq\|x\|
$$

which proves the assertion.

Proposition 2. For any value of $\mu, B_{\infty} \subset \sigma(H)$ and there is no eigenvalue in $B_{\infty}$.

Proof. Let $\psi \neq 0$ be a solution of $(H-E) \psi=0$. Proposition 1 implies that $\Psi_{2 F_{n}}=M^{2}(n) \Psi_{0}$ and $\Psi_{-2 F_{n}-2}=L^{2}(n) \Psi_{-2}$ for $n \geqq 3$. Let $E \in B_{\infty}$; then $\left|x_{n}\right| \leqq c$ for all $n$, with some $c<\infty$. Apply Lemma 1 , at first, with $B=M(n)$ and $x=\Psi_{0}$, and at second, with $B=L(n)$ and $x=\Psi_{-2}$. This yields for $n \geqq 3$,

$$
\begin{aligned}
& \max \left\{2 c\left\|\Psi_{F_{n}}\right\|,\left\|\Psi_{2 F_{n}}\right\|\right\} \geqq \frac{1}{2}\left\|\Psi_{0}\right\|, \\
& \max \left\{2 c\left\|\Psi_{-F_{n}-2}\right\|,\left\|\Psi_{-2 F_{n}-2}\right\|\right\} \geqq \frac{1}{2}\left\|\Psi_{-2}\right\| .
\end{aligned}
$$

Therefore, $E$ is not an eigenvalue but it is in the spectrum. Indeed, suppose that $E \notin \sigma(H)$. Then there is a unique $\psi \in l^{2}(\mathbb{Z})$ which solves the equations $((H-E) \psi)(k)$ $=\delta_{k, 0}$. For $k \neq 0$ these are homogeneous, hence $\psi$ satisfies (11). At least one of $\psi(-1)$, $\psi(0)$ and $\psi(1)$ is nonzero, therefore one of $\Psi_{0}$ and $\Psi_{-2}$ is nonzero, and (11) contradicts $\psi \in l^{2}$.

In order to show $B_{\infty}=\sigma(H)$, we need to study $B_{\infty}^{c}=\mathbb{R}-B_{\infty}$. 
Lemma 2. Consider the sequence $x_{-1}=1, x_{0}, x_{1}, \ldots$, generated by the iteration $x_{n+2}=2 x_{n+1} x_{n}-x_{n-1}$. A sufficient and necessary condition that $\left\{x_{n}\right\}$ be unbounded is that

$$
\left|x_{N-1}\right| \leqq 1, \quad\left|x_{N}\right|>1 \text { and }\left|x_{N+1}\right|>1
$$

for some $N \geqq 0$. This $N$ is unique, $\left|x_{n+2}\right|>\left|x_{n+1} x_{n}\right|>1$ for $n \geqq N$, and there exists $c>1$ such that $\left|x_{n}\right|>c^{F_{n-N}}$. If $\left\{x_{n}\right\}$ is bounded then $\left|x_{n}\right|<1+\left|x_{1}-x_{0}\right|$, any $n$.

Remark. The lemma implies that $\left|x_{n}\right|>\left|x_{n-1}\right|, n \geqq N+2$.

Proof. Suppose that (12) holds true with some $N \geqq 0$. Then $\left|x_{N+2}\right| \geqq\left|x_{N+1} x_{N}\right|+$ $\left(\left|x_{N+1} x_{N}\right|-\left|x_{N-1}\right|\right)>\left|x_{N+1} x_{N}\right|>1$, and by induction we get $\left|x_{n+2}\right|>\left|x_{n+1} x_{n}\right|$ for any $n \geqq N . \log \left|x_{n+2}\right|>\log \left|x_{n+1}\right|+\log \left|x_{n}\right|$ shows that $\log \left|x_{n}\right|$ increases faster than the Fibonacci sequence, whence $\left|x_{n}\right|>c^{F_{n-N}}, n \geqq N$. We found that

$$
\left|x_{n-1}\right| \leqq 1<\left|x_{n}\right|,\left|x_{n+1}\right|<\left|x_{n+2}\right|<\left|x_{n+3}\right|<\cdots
$$

if $n=N$; clearly these inequalities cannot hold for other values of $n$. Suppose now that (12) is not valid for any $N$, and let $\left|x_{n}\right|>1$. Then $\left|x_{n-1}\right| \leqq 1$ and $\left|x_{n+1}\right| \leqq 1$ (and, in fact, $\left|x_{n-1} x_{n+1}\right|<1$ ), otherwise we would get (12) with $N \leqq n$. From (10),

$$
\left|x_{n}\right| \leqq\left|x_{n-1} x_{n+1}\right|+\left(\left(1-x_{n-1}^{2}\right)\left(1-x_{n+1}^{2}\right)+I\right)^{1 / 2}
$$

and the maximum of the rhs, with the condition that $\left|x_{n-1}\right| \leqq 1,\left|x_{n+1}\right| \leqq 1$, is at $\left|x_{n-1}\right|=\left|x_{n+1}\right|=1$. This yields $\left|x_{n}\right|<1+\sqrt{I}$, as claimed.

For fixed $\mu$, define

$$
\rho_{n}=\left\{E \in \mathbb{R}|| x_{n} \mid>1\right\}, \quad \sigma_{n}=\left\{E \in \mathbb{R}|| x_{n} \mid \leqq 1\right\} .
$$

Here $x_{n}(E)$ are polynomials of order $F_{n}$, as it can be seen from Eqs. (2), (3) and (9). In particular, $x_{0}=E / 2$ and $x_{1}=(E-\mu) / 2$. Obviously $\rho_{n}$ are open sets. We have

Proposition 3. $B_{\infty}^{c}$ is an open set, and

$$
B_{\infty}^{c}=\bigcup_{n=N}^{\infty}\left(\rho_{n} \cap \rho_{n+1}\right), \quad \text { any } N \geqq 0 .
$$

Moreover,

$$
\rho_{n} \cap \rho_{n+1}=\bigcap_{k=n}^{\infty} \rho_{k}, \quad \text { any } n \geqq 0 .
$$

Proof. $B_{\infty}$ is closed, due to the uniform bound $\left|x_{n}(E)\right|<1+|\mu| / 2$ for $E \in B_{\infty}$ and the continuity of $x_{n}(E)$. From Lemma 2,

$$
B_{\infty}^{c}=\left(\rho_{0} \cap \rho_{1}\right) \cup\left(\bigcup_{n=1}^{\infty}\left(\sigma_{n-1} \cap \rho_{n} \cap \rho_{n+1}\right)\right)
$$

is a disjoint decomposition of $B_{\infty}^{c}$. Clearly,

$$
\rho_{n} \cap \rho_{n+1}=\left(\rho_{0} \cap \rho_{1}\right) \cup\left(\bigcup_{k=1}^{n}\left(\sigma_{k-1} \cap \rho_{k} \cap \rho_{k+1}\right)\right), \quad n \geqq 0,
$$


hence we find

$$
\rho_{n} \cap \rho_{n+1} \subset \rho_{n+1} \cap \rho_{n+2}
$$

and Eq. (14). $\rho_{n} \cap \rho_{n+1} \subset \bigcap_{k=n}^{\infty} \rho_{k}$ is a consequence of Lemma 2, and the inclusion in the other sense is trivial.

In what follows, we construct periodic approximations to $H$. The notation $\rho(A)$ is used for the intersection of the resolvent set of the operator $A$ with the real axis.

Proposition 4. Let $\left\{H_{m}\right\}_{m=1}^{\infty}$ be a sequence of Schrödinger operators on $l^{2}(\mathbb{Z})$ given by

where

$$
\left(H_{m} \psi\right)(n)=\psi(n+1)+\psi(n-1)+\mu v_{m}(n) \psi(n)
$$

$$
v_{m}(n)=\left[(n+1) \omega_{m}\right]-\left[n \omega_{m}\right], \quad \omega_{m}=F_{m-1} / F_{m} .
$$

Then $H=\mathrm{s}-\lim H_{m}$, and $\rho\left(H_{m}\right)=\rho_{m}$.

Proof. Exploiting the fact that $\omega_{m}$ is a best approximation to $\omega$, one easily verifies that

$$
v_{m}(n)=v(n) \text { for } \begin{cases}-F_{m}+1 \leqq n \leqq F_{m}+1, & m \geqq 2 \text { even } \\ -F_{m-1}+1 \leqq n \leqq F_{m-2}, & m \geqq 3 \text { odd } .\end{cases}
$$

Take $\psi \in l^{2}(\mathbb{Z})$; for $m \geqq 3$,

$$
\left\|\left(H-H_{m}\right) \psi\right\|^{2}=\mu^{2} \sum_{|n| \geqq F_{m-2}}\left(v(n)-v_{m}(n)\right)^{2}|\psi(n)|^{2} \leqq \mu^{2} \sum_{|n| \geqq F_{m-2}}|\psi(n)|^{2} \rightarrow 0,
$$

which proves that $H$ is the strong limit of $\left\{H_{m}\right\}$. Now $v_{m}$ is periodic with period $F_{m}$. According to (17), for $m \geqq 2$ even $v_{m}$ repeats periodically the segment $\left(v(1), \ldots, v\left(F_{m}\right)\right.$ ), therefore the transfer matrix over a period is $M(m)$. For $m \geqq 3$ odd, $v_{m}$ repeats periodically the segment $\left(v\left(-F_{m-1}+1\right), \ldots, v\left(F_{m-2}\right)\right)$. Due to (7), this coincides with $\left(v(1), \ldots, v\left(F_{m}\right)\right)$, therefore $M(m)$ can be chosen to be the transfer matrix over a period. Since $|\operatorname{Tr} M(m)| \leqq 2$ is sufficient and necessary that $E \in \sigma\left(H_{m}\right)$, the spectrum of $H_{m}$, we find $\sigma\left(H_{m}\right)=\sigma_{m}$ and $\rho\left(H_{m}\right)=\rho_{m}$.

In the following lemma $U^{\circ}$ denotes the interior of the set $U$.

Lemma 3. Let $A,\left\{A_{m}\right\}$ be bounded self-adjoint operators on a Hilbert space, $A=$ s- $\lim A_{m}$. Then

$$
\left(\bigcap \rho\left(A_{m}\right)\right)^{\circ} \subset \rho(A) \text {. }
$$

Remark. The lemma remains valid if $\rho$ is replaced by the whole resolvent set.

Proof. Let $E \in\left(\bigcap \rho\left(A_{m}\right)\right)^{\circ} \neq \varnothing$. Then there exists $K<\infty$ such that $\left\|\left(A_{m}-E\right)^{-1}\right\|$ $\leqq K$. Therefore, for any vector $\phi$ we have $\left\|\left(A_{m}-E\right) \phi\right\| \geqq\|\phi\| / K$, and, taking the limit $m \rightarrow \infty,\|(A-E) \phi\| \geqq\|\phi\| / K$. By Weyl's criterion, this means $E \in \rho(A)$.

Proof of Theorem 1. After Proposition 2, it remained to show that $B_{\infty}^{c} \subset \rho(H)$. Take $E \in B_{\infty}^{c}$; by Propositions 3 and 4 there exists some $n \geqq 0$ such that 
$E \in \rho\left(H_{n}\right) \cap \rho\left(H_{n+1}\right)=\left(\bigcap_{k=n}^{\infty} \rho\left(H_{k}\right)\right)^{\circ}$. Since $H=$ s-lim $H_{k}$, Lemma 3 applies, whence $E \in \rho(H)$.

Combining Theorem 1 with Proposition 3 one immediately obtains

Proposition 5. $\sigma_{n} \cup \sigma_{n+1}$ is monotonically decreasing, and tends to $\sigma(H)$, i.e., $\sigma_{n} \cup$ $\sigma_{n+1} \supset \sigma_{n+1} \cup \sigma_{n+2}$, and $\sigma(H)=\bigcap_{n=N}^{\infty}\left(\sigma_{n} \cup \sigma_{n+1}\right)$, any $N \geqq 0$.

The spectrum is nonempty, closed, and from Theorem 1 we know that it does not contain isolated points. We can complete the

Proof of Theorem 2. From Eqs. (9), (10) and (13) it follows that for any $n \geqq 0$, $\sigma_{n} \cap \sigma_{n+1} \cap \sigma_{n+2}=\varnothing$ if $I>4$, and $\sigma_{n} \cap \sigma_{n+1} \cap \sigma_{n+2} \cap \sigma(H)=\varnothing$ if $I=4$. Fix $|\mu| \geqq 4$; then $I \geqq 4$. Now $\sigma(H)$ is a Cantor set if $\sigma(H)^{\circ}=\varnothing$. Suppose the opposite; then there exists an open interval $\Lambda \subset \sigma(H) . \Lambda \subset \rho_{n}$ cannot occur for an infinite number of indices: otherwise $\Lambda \subset\left(\bigcap_{k=1}^{\infty} \rho_{n_{k}}\right)^{\circ} \subset \rho(H)$ would be contradictory. Hence, there exists $N(\Lambda)$ such that $\sigma_{n} \cap \Lambda \neq \varnothing$ if $n \geqq N(\Lambda)$. Fix $n>N(\Lambda) ; \Lambda \subset \sigma_{n} \cap \sigma_{n+1} \cap \sigma_{n+2}$ cannot hold, so that for some $m \in\{n, n+1, n+2\} \sigma_{m}$ has a boundary point $E_{0}$ in $\Lambda$. Since $\Lambda \subset \sigma_{m-1} \cup \sigma_{m}$ and $\Lambda \subset \sigma_{m} \cup \sigma_{m+1}, E_{0}$ is the accumulation point of both $\sigma_{m-1}$ and $\sigma_{m+1}$. All the $\sigma_{i}$ are closed, hence $E_{0} \in \sigma_{m-1} \cap \sigma_{m} \cap \sigma_{m+1}$; but this is impossible for $|\mu| \geqq 4$.

Remark. An alternate proof can be obtained by observing that $I>4$ and $E_{0} \in \sigma(H)$ imply $E_{0} \in \bigcap_{k=1}^{\infty}\left(\sigma_{n_{k}-1} \cap \rho_{n_{k}} \cap \sigma_{n_{k}+1}\right)$, where $n_{k+1}-n_{k}=2$ or 3 , and $1<\sqrt{I}-1$ $\leqq\left|x_{n_{k}}\left(E_{0}\right)\right|<\sqrt{I}+1$ (cf. (10)). Suppose that $E_{0} \in \Lambda \subset \sigma(H)$ for some open interval $\Lambda$. By the continuity of $x_{n_{k}}(E)$, the sequence $\left\{n_{k}\right\}$ must be the same for all $E \in \Lambda$, therefore $\Lambda \subset\left(\bigcap_{k=1}^{\infty}\left(\sigma_{n_{k}-1} \cap \rho_{n_{k}} \cap \sigma_{n_{k}+1}\right)\right)^{\circ} \subset\left(\bigcap_{k=1}^{\infty} \rho_{n_{k}}\right)^{\circ} \subset \rho(H)$, which is a contradiction.

Acknowledgements. I am indebted to J. Bellissard, J.-J. Loeffel and Ch.-E. Pfister for many helpful discussions. This work was supported by the Fonds National Suisse through Grant No. 2.683-085.

\section{References}

1. Kohmoto, M., Kadanoff, L. P., Tang, Ch.: Localization problem in one dimension: Mapping and escape. Phys. Rev. Lett. 50, 1870-1876 (1983)

2. Ostlund, S., Pandit, R., Rand, D., Schellnhuber, H. J., Siggia, E. D.: One-dimensional Schrödinger equation with an almost periodic potential. Phys. Rev. Lett. 50, 1873-1876 (1983)

3. de Bruijn, N. G.: Sequences of zeros and ones generated by special production rules. Indagationes Math. 84, 27-37 (1981)

4. Rand, D., Ostlund, S., Sethna, J., Siggia, E. D.: Universal transition from quasiperiodicity to chaos in dissipative systems. Phys. Rev. Lett. 49, 132-135 (1982)

5. Feigenbaum, M. J., Hasslacher, B.: Irrational decimations and path integrals for external noise. Phys. Rev. Lett. 49, 605-609 (1982)

6. Kohmoto, M., Oono, Y.: Cantor spectrum for an almost periodic Schrödinger equation and a dynamical map. Phys. Lett. 102A, 145-148 (1984) 
7. Casdagli, M.: Symbolic dynamics for the renormalization map of a quasiperiodic Schrödinger equation. Commun. Math. Phys. 107, 295-318 (1986)

8. Lang, S.: Introduction to diophantine approximations. Reading, MA: Addison-Wesley 1966 Chap. 1

9. Simon, B.: Almost periodic Schrödinger operators: A review. Adv. Appl. Math. 3, 463-490 (1982)

10. Delyon, F., Petritis, D.: Absence of localization in a class of Schrödinger operators with quasiperiodic potential. Commun. Math. Phys. 103, 441-444 (1986)

Communicated by B. Simon

Received December 2, 1986 
